\title{
Bilateral Leukemic Pulmonary Infiltrates: An Initial Presentation of Acute Lymphoblastic Leukemia Relapse
}

\author{
Kushagra Taneja $^{1}$ (1) $\cdot$ Chandrika Verma $^{1} \cdot$ Amita Mahajan $^{1}$
}

Received: 16 February 2021 / Accepted: 1 July 2021 / Published online: 23 July 2021

c) Dr. K C Chaudhuri Foundation 2021

Leukemic lung infiltrates are known in acute myeloid leukemia [1] and have occasionally been described in pediatric acute lymphoblastic leukemia (ALL) [2]. Pulmonary complications in acute leukemia may be infectious or noninfectious. The latter includes pulmonary hemorrhage, edema, embolism, and leukostasis. Late relapse in pediatric ALL presenting as pulmonary leukemic infiltrates has not been reported in literature.

A relapsed ALL case with pulmonary infiltrates at initial presentation is presented here. The boy had been treated for B-ALL $10 \mathrm{y}$ ago. He presented to a general practitioner with fever, weight loss, and dyspnea for 3 wk. Chest radiograph revealed bilateral diffuse nodular opacities (Fig. 1a). The differentials could be infective (tuberculosis, fungal pneumonia) and noninfective (malignancy, metastasis, and hypersensitivity pneumonitis). CBC showed mild neutrophilic leukocytosis (Hb-12.5/TLC-12,500/Platelet-180000]. He was empirically given antitubercular treatment but there was clinical worsening in $2 \mathrm{wk}$. COVID-19 RT-PCR was negative thrice.

High-resolution computed tomography (HRCT) of the chest revealed diffuse pulmonary nodules with ground glass opacities (GGO) (Fig. 1b), atypical of COVID, which is characterized by peripheral and subpleural GGOs and consolidations. Repeat CBC showed leukocytosis with 92\% blasts. Bronchoalveolar lavage showed lymphoblasts. Flow cytometry from peripheral blood confirmed B-ALL.

Chemotherapy was commenced as per relapse protocol. Repeat chest radiograph and HRCT at 4 wk showed complete resolution (Fig. 1c, d). This highlights the rare presentation of late ALL relapse with primary pulmonary involvement, which needs to be differentiated from common infective etiologies.

Kushagra Taneja

kushagrataneja321@gmail.com

1 Department of Pediatric Hematology-Oncology, Indraprastha Apollo Hospital, New Delhi 110076, India

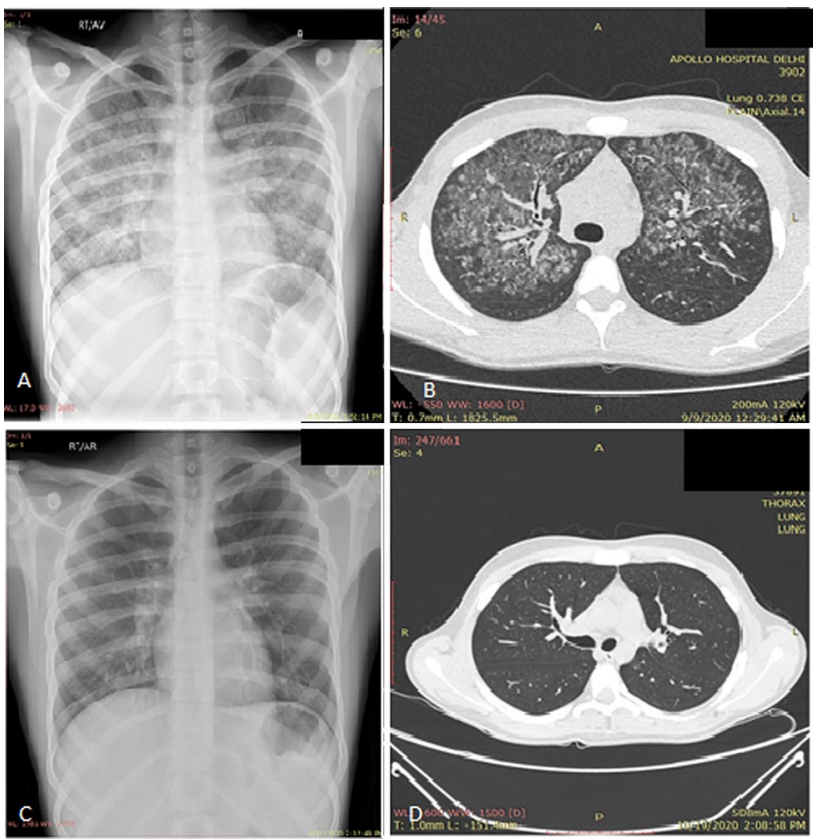

Fig. 1 Chest radiograph PA view and HRCT axial section at presentation showing bilateral diffuse opacities ( $\mathbf{A}$ and $\mathbf{B})$, and after $4 \mathrm{wk}$ of treatment showing clearance of visualized lung fields $(\mathbf{C}$ and $\mathbf{D})$

\section{Declarations}

Consent Exemption The data reproduced in the given case report consists of radiological images which do not reveal the identity of the patient.

Conflict of Interest None.

\section{References}

1. Guermazi A, Feger C, Rousselot P, et al. Granulocytic sarcoma (chloroma): imaging findings in adults and children. AJR American J Roentgenol. 2002;178:319-25.

2. Kelleher JF Jr, Miale TD, Donnelly WH. Respiratory distress secondary to pulmonary leukemia. Am J Dis Child. 1981;135:716-8.

Publisher's Note Springer Nature remains neutral with regard to jurisdictional claims in published maps and institutional affiliations. 